\title{
Laurent Feneyrou, De lave et de fer. Une jeunesse allemande: Helmut Lachenmann
}

Paris, éditions MF, 2017

\section{Laetitia Devos}

\section{(2) OpenEdition}

\section{Journals}

Édition électronique

URL : http://journals.openedition.org/transposition/2901

DOI : $10.4000 /$ transposition.2901

ISSN : 2110-6134

\section{Éditeur}

CRAL - Centre de recherche sur les arts et le langage

\section{Référence électronique}

Laetitia Devos, «Laurent Feneyrou, De lave et de fer. Une jeunesse allemande : Helmut Lachenmann», Transposition [En ligne], 8 | 2019, mis en ligne le 15 septembre 2019, consulté le 17 décembre 2020. URL : http://journals.openedition.org/transposition/2901 ; DOI : https://doi.org/10.4000/transposition. 2901

Ce document a été généré automatiquement le 17 décembre 2020.

\section{(c) (i) (2)}

La revue Transposition est mise à disposition selon les termes de la Licence Creative Commons Attribution - Partage dans les Mêmes Conditions 4.0 International. 


\section{Laurent Feneyrou, De lave et de fer. Une jeunesse allemande: Helmut Lachenmann}

Paris, éditions MF, 2017

\section{Laetitia Devos}

\section{RÉFÉRENCE}

Laurent Feneyrou, De lave et de fer. Une jeunesse allemande : Helmut Lachenmann, Paris, éditions MF, 2017

1 C'est à une approche inédite de l'esthétique de Helmut Lachenmann que Laurent Feneyrou invite, en nous immergeant dans le climat politique, esthétique et intellectuel entourant des œuvres majeures $\mathrm{du}$ compositeur. Il procède en trois «coupes franches ", qui sont matérialisées par des pages noires, à la fois repères pratiques pour le lecteur, mais peut-être aussi suggestion métaphorique du trou noir ouvert par la vague terroriste : 1967, 1977 et 1997.

2 Sans fausse promesse, le sous-titre «Une jeunesse allemande: Helmut Lachenmann » annonce qu'il va être question, outre du compositeur né en 1935, de toute une génération allemande, la "jeunesse » de 1968. On compte parmi elle Gudrun Ensslin (1940-1977), une des figures centrales de la Fraction armée rouge ainsi que de cet ouvrage, amie d'enfance du compositeur et inspiratrice de la « litanie » de La Petite Fille aux allumettes. Le titre « De lave et de fer » est justement une expression empruntée à une de ses lettres écrites en prison et qui évoque la seule alternative possible à la mort (" soit "on" crève, soit des gens comme nous deviennent définitivement de la lave ou du fer », p. 110).

3 L. Feneyrou étudie un nombre de sources et d'études si important qu'il a dû (malheureusement) renoncer à une bibliographie en fin d'ouvrage, la foisonnante documentation étant déjà indiquée en notes. L'un des mérites de cet essai - parmi tant 
d'autres - est de proposer une traduction française, nouvelle ou revue, d'un grand nombre de sources jusque-là peu ou pas accessibles à des non-germanistes. Les écrits de Helmut Lachenmann, traduits aux éditions Contrechamps sous la direction de Martin Kaltenecker, sont enrichis de remarques traductologiques, notamment au sujet de termes comme wehrlos (p. 159), Abschattung (p. 152) ou abtasten (p. 150).

4 L'originalité méthodologique consiste à accorder une grande place au contexte politicoidéologique sans toutefois forcer l'interprétation de l'œuvre dans ce sens. Ouvrage tout à la fois d'histoire, d'esthétique et de musicologie, ce sont des concepts philosophiques, comme celui du refus ou celui du toucher, qui font le lien entre les différents champs.

5 Les deux premiers tiers de la première partie, intitulée sobrement « 1967 », retracent la naissance de la protestation étudiante en Allemagne de l'Ouest. Celle-ci naît au milieu des années 1950, lors du réarmement du pays. L. Feneyrou prend le parti de faire entendre uniquement les voix contestataires et de ne pas entrer dans les questions de droit international liées au recouvrement de la souveraineté de la RFA en 1955. Il en va de même pour la loi sur l'état d'exception réintroduite dans le droit allemand en juin 1968 : sans évoquer les justifications officielles et juridiques, l'auteur donne la parole à ceux qui s'alarment de ce qui rappelle les heures sombres de l'histoire allemande. «Une jeunesse allemande » découvre avec horreur la responsabilité parentale sous le nazisme, à l'image de Bernward Vesper (compagnon de Gudrun Ensslin).

6 Sans doute mieux connue des lecteurs français, la protestation contre la guerre du Vietnam est aussi l'un des fers de lance de l'Union des étudiants socialistes allemands (Sozialistischer Deutscher Studentenbund). L. Feneyrou montre l'émergence des "Communes» et convoque une impressionnante quantité de sources, précisant de surcroît en notes celles qui se trouvaient dans la bibliothèque personnelle de Luigi Nono, auprès de qui Lachenmann étudia et séjourna de 1958 à 1960.

7 Un récit retrace les événements qui conduisent à la mort de l'étudiant Benno Ohnesorg le 2 juin 1967, tué par un policier. La presse du groupe Springer se déchaîne contre les étudiants et les intellectuels, au rang desquels G. Grass, H. Marcuse, Th. W. Adorno ou H. Böll : L. Feneyrou présente leurs thèses avec tout à la fois exhaustivité et esprit de synthèse, nous plongeant dans le débat de l'époque.

8 Le lien avec la scène musicale se fait en septembre 1967, lorsque des contestataires, dont Gudrun Ensslin et Andreas Baader, font irruption dans un concert dirigé par Pierre Boulez à Berlin (p. 35 sq.). Puis en février 1968, Werner Henze et Luigi Nono participent à l'organisation du congrès international pour le Vietnam à Berlin-Ouest. Enfin, Rudi Dutschke, après l'attentat dont il est victime le 11 avril 1968, passe plusieurs mois de convalescence chez Henze. L'incendie de grands magasins de Francfort en avril 1968 signe le basculement vers une résistance qui assume désormais son appel à la violence, comme l'explicitent des articles d'Ulrike Meinhof amplement cités. C'est dans ce contexte qu'a lieu la création, ou plutôt la non-création, du Radeau de la Méduse de Henze le 9 novembre 1968 à Hambourg, empêchée par un affrontement, tant dans la salle que sur le plateau, entre des militants de gauche et leurs opposants. Après une présentation de l'argument du Radeau de la Méduse, allégorie du combat du TiersMonde, et de sa musique, Feneyrou s'intéresse à une œuvre de Lachenmann qui en est contemporaine : Air pour grand orchestre avec percussion soliste, créé le $1^{\mathrm{er}}$ septembre 1969. Dans un paragraphe placé en retrait pour avertir le lecteur qu'il s'agit d'une analyse musicologique plus poussée, l'auteur détaille cinq mesures, considérées comme 
le point culminant de l'œuvre et dont la partition figure en regard. L'auteur n'en déduit pas d'interprétation unilatéralement esthétique ou idéologique :

Il ne s'agit pas ici d'un art d'agit-prop imitant ou singeant les manifestations étudiantes et leur répression, mais du socle commun d'une réalité acoustique déployant les plans du matériau, de la structure musicale et de l'aura partant de l'histoire. Et jamais l'un sans l'autre. À exclure le matériau et la structure, on dénature la musique en mésestimant sa rigueur; à exclure l'aura et l'histoire, on en oublie l'expérience politique et historique. Toute la tension de l'œuvre de Lachenmann est là. (p. 54)

9 Avec un sens pédagogique très appréciable, L. Feneyrou fait l'exégèse des écrits théoriques de Lachenmann en cette fin des années 1960. On retient notamment un «bréviaire » du concept du « refus» (p. 61 sq.), que le compositeur a repris à Marcuse, et trop souvent galvaudé par la critique, ainsi que des explications sur l'«aura» (p. 70-71). Par cette navigation entre textes théoriques et œuvres, parmi lesquelles Pression pour violoncelle (1969), l'auteur nous introduit dans le débat entre Lachenmann et Nono, entre Lachenmann et l'avant-garde sérielle, et enfin entre Lachenmann et Henze, dont la polémique de 1982 (l'auteur s'accordant une prolepse qui, ainsi, évite l'écueil d'une présentation chronologique trop linéaire) conclut la première partie.

10 La deuxième partie est, tout aussi modestement que la première, intitulée «1977 » et suit le même schéma : les deux premiers tiers sont consacrés à une remise en contexte et le dernier tiers aux œuvres elles-mêmes. Le récit de la radicalisation des protestataires entre 1968 et cette année 1977 se fait par jalons: ceux des actions terroristes elles-mêmes bien sûr (qui culminent lors de «l'automne allemand »), mais aussi des théories qui les sous-tendent, notamment celle de la guérilla urbaine (p. 87-97). Le lien avec la première partie de l'ouvrage nous semble suggéré entre les lignes, l'auteur accordant de nouveau un développement à l'idée du « refus » (p. 89), mais cette fois dans le sens où l'entendent les contestataires radicaux, et sans tomber dans une analogie qui aurait été trop directe avec la théorie de Lachenmann.

11 L'auteur cite amplement, voire in extenso, les communiqués des commandos terroristes (p. 107-108) ou de sympathisants (p. 123-127). Il accorde donc une large place à la question de l'articulation entre la violence de la guérilla et la (supposée) violence d'État, là où d'autres n'y voient que l'expression d'esprits «malades ». Laissant le lecteur se forger sa propre opinion, L. Feneyrou n'intervient que très ponctuellement pour condamner, dans ces écrits de la gauche radicale, les amalgames qui lui semblent dangereux, par exemple entre la guerre du Vietnam, les camps nazis et les bombardements alliés de la fin de la Deuxième Guerre mondiale sur Dresde et Hambourg (p. 108) ou encore dans les positions à l'égard d'Israël (p. 29 et p. 111).

12 Pour les artistes, la question est de savoir « comment répondre à un tel déferlement de violence.» (p. 108) L'auteur analyse alors des réponses venues d'Italie, qui traversait une crise semblable : celle de Nono, dans Al gran sole carico d'amore (1972-1974, révision en 1977), dont le quatuor vocal est mis en regard de celui employé en 1974 par Giacomo Manzoni dans Per Massimiliano Robespierre (p. 100-101).

13 Quant à Lachenmann, au plus fort de la crise de l'année 1977, il compose d'avril à octobre Salut für Caudwell, « musique pour deux guitaristes ». Le compositeur lui-même soutient la thèse d'un lien direct entre l'œuvre et le contexte, affirmant que « l'appel à la société [...] mais aussi le gestus corporel de l'écrivant instrumental [...] invoquaient, 
avant même la mort de Gudrun Ensslin qu'[il] connaissait depuis l'enfance, l'ultime situation, sans langage, de l'esprit sensibilisé par la politique »(p. 138). L Feneyrou voit dans Salut für Caudwell « quelque chose de l'ordre de la discussion, de la dispute ou du démêlé entre le musical et le politique » (p. 138), à l'image de la reprise de l'exhortation du troisième livre de Zarathoustra de Nietzsche « Mensch gib acht!», à comprendre au double sens de « prendre garde » et « écouter ».

C'est ensuite la Tanzsuite mit Deutschlandlied (1979-1980) qui est présentée, avant que L. Feneyrou ne propose une lecture commune des deux œuvres par le prisme notionnel du «toucher »: le détour sur la tradition «tactiliste» de la philosophie s'avère particulièrement éclairant pour comprendre l'apport nouveau de Lachenmann à la relation entre le toucher et l'écoute. La boucle est bouclée lorsque l'auteur file la métaphore du "corps politique » et partant, de "l'orthopédie sociale " (à laquelle s'opposent les grèves de la faim). Le «toucher" de Lachenmann est finalement vu comme « lieu d'émergence d'un contre-pouvoir, [...] un moment de libération » (p. 158).

La troisième partie, "1997 », se concentre sur La Petite Fille aux allumettes, " musique avec images ", créée en janvier de cette même année à Hambourg, et que L. Feneyrou caractérise d'emblée de « réponse musicale à la violence de l'automne allemand [...] des années plus tard, toujours à travers la question du corps et de la biopolitique » (p. 165). Après une présentation de la genèse, puis de l'œuvre elle-même qui partage avec Salut für Caudwell l'usage du «morse augmenté », la « litanie », sur une lettre d'Ensslin citée dans son intégralité ici, est analysée à la lumière de l'essai du psychanalyste Didier Anzieu sur le "Moi-peau» (p.173-178). Parmi les tortures "blanches", celle de l'isolement acoustique recueille tout particulièrement l'attention de l'auteur. L'étouffement du son, déjà menaçant dans Salut für Caudwell, trouve son équivalent dans La Petite Fille aux allumettes, «le chœur chuchotant presque le paragraphe de la lettre d'Ensslin, [...] [avec une] ligne rythmique gorgée de silences » (p. 182).

L. Feneyrou reprend finalement la question présentée en avant-propos, celle de savoir si H. Lachenmann exprime dans cette litanie une « donnée biographique essentielle ", thèse que semble nourrir la déclaration suivante du compositeur au sujet d'Ensslin : « Il n'y a aucune excuse pour ses actions criminelles. Mais en les condamnant, on ne règle pas la question de notre co-responsabilité. » Plus largement, l'auteur se demande si une œuvre musicale comme celle-ci procède de l'«historicisation de la Fraction armée rouge " à une heure où cette dernière a prononcé sa dissolution. Plaide en ce sens une liste impressionnante, mais déclarée non exhaustive, d'une soixantaine d'artistes qu'il n'avait pas encore cités et venant compléter les seize "excursus » disséminés dans l'ouvrage - synthèses et commentaires de films, vidéos d'art, installations, pièces de théâtre et œuvres plastiques inspirés par ces années de plomb.

Enfin, L. Feneyrou convoque les thèses sur l'histoire de Walter Benjamin, invoquées par les membres de la Fraction Armée Rouge eux-mêmes pendant leur procès, dont il montre l'interprétation dévoyée. Si l'auteur revient sur ce qui oppose le philosophe à la Théologie politique de Schmitt, c'est aussi parce que L'origine du drame baroque a été une des références du Prometeo de Nono ("tragédie de l'écoute», 1981-1985), œuvre qui elle-même est le reflet de questionnements sur la légitimité de la violence et critique d'une présentation lisse et causale de l'histoire.

Un projet de Nono sur Meinhof et ses privations sensorielles en prison, resté à l'état d'esquisse, sert d'entrée dans la section 23 ( «Shō») de La Petite Fille aux allumettes. Cette section est aussi analysée à l'aune de la philosophie de Kitaro Nishida qui a lui-même 
été une source de Lachenmann dans Nun (Maintenant, 1997-1999, supplément à La Petite fille aux allumettes). Pour Nishida, le moi est « le lieu en lequel le son devient manifeste en tant que tel»(p.216). La Petite Fille aux allumettes et Nun participent, selon une synthèse que L. Feneyrou reprend à $\mathrm{M}$. Kaltenecker, de la "troisième manière " de Lachenmann, dans laquelle «le sujet et le lieu sont désormais un ». Feneyrou conclut par une exégèse du texte " La musique est morte » (1996) et par une topologie du néant, le compositeur ayant "radicalisé l'abîme de la nihilité »: cette dernière n'est plus le « refus » de la fin des années 1960, mais « l'identité à soi absolument contradictoire, du “est et n'est pas", de l'être en tant que néant et du néant en tant qu'être » (p. 223). Ainsi, dans Nun et dans la section Shō de La Petite Fille aux allumettes, " le son disparaît dans son apparition même» (p. 223). De la même manière, le temps "disparaît en chacun de ses instants, touchant là au néant absolu et au maintenant éternel.» (p. 227-228) La rupture de ce temps lisse a déjà été précédemment rapprochée par l'auteur de la rupture temporelle des actes terroristes.

L. Feneyrou défend la thèse que la négation n'est pas annulation chez Lachenmann et invite « à repenser le statut du négatif dans son œuvre » (p. 215). L'ouvrage s'achève, ou plutôt s'ouvre sur une citation de Nishida et sur la question « et maintenant?» (Nun), laissant le lecteur continuer son cheminement dans l'œuvre du compositeur, enrichi de la lecture de cet essai foisonnant.

\section{AUTEURS}

\section{LAETITIA DEVOS}

Laetitia Devos est maître de conférences en études germaniques à l'université Rennes 2 où elle enseigne la langue et la civilisation allemandes depuis 2009. Également titulaire d'une licence de musicologie de l'université Paris IV, ses recherches portent sur les liens entre musique, littérature et contexte politique au $\mathrm{xx}^{\mathrm{e}}$ siècle. Outre différents articles, elle a publié l'ouvrage L'opéra en RDA. Sous le signe de Georg Büchner aux Presses universitaires de Rennes (collection Le Spectaculaire) en 2012. 